\title{
Oligomeric Silica-Wrapped Perovskites Enable Synchronous Defect Passivation and Grain Stabilization for Efficient and Stable Perovskite Photovoltaics
}

Yang Bai, ${ }^{\dagger, \S \odot ~ Y u n ~ L i n, ~}{ }^{\dagger, \ddagger \odot}$ Long Ren,, Xiaolei Shi, ${ }^{\perp} \odot$ Ekaterina Strounina, ${ }^{*}$ Yehao Deng, ${ }^{\dagger \dagger}$ Qi Wang, ${ }^{\dagger, \ddagger}$ Yanjun Fang, ${ }^{\dagger}$ Xiaopeng Zheng, ${ }^{\dagger}$ Yuze Lin, ${ }^{\dagger,}$ Zhi-Gang Chen, ${ }^{\perp, \nabla_{\odot}}$ Yi Du, ${ }^{\|}$Lianzhou Wang, and Jinsong Huang*,

${ }^{\dagger}$ Department of Mechanical and Materials Engineering, University of Nebraska-Lincoln, Lincoln, Nebraska 68588, United States ${ }^{\ddagger}$ Department of Applied Physical Science, University of North Carolina, Chapel Hill, North Carolina 27514, United States

${ }^{\S}$ Nanomaterials Centre, Australian Institute for Bioengineering and Nanotechnology and School of Chemical Engineering, The University of Queensland, St Lucia, Queensland 4072, Australia

"Institute for Superconducting and Electronic Materials (ISEM), Australian Institute for Innovative Materials (AIIM), University of Wollongong, Wollongong, New South Wales 2500, Australia

${ }^{\perp}$ Materials Engineering, The University of Queensland, St Lucia, Queensland 4072, Australia

${ }^{\#}$ Centre for Advanced Imaging, The University of Queensland, Brisbane, Queensland 4072, Australia

${ }^{\nabla}$ Centre for Future Materials, University of Southern Queensland, Springfield, Queensland 4300, Australia

ABSTRACT: The intrinsic instability of hybrid perovskite materials induced by defect states arises as one major challenge hampering the commercialization of perovskite solar cells (PSCs). Here, we report a facile strategy of wrapping perovskite grains within an oligomeric silica (OS) matrix in a core-shell geometry, which can synchronously passivate the defects at surfaces and grain boundaries and stabilize the grains at the nanoscale. We observe a significant reduction of trap density and elongation of carrier lifetime in OS-wrapped perovskites, which yields an increased efficiency of $21.5 \%$ for $p-i-n$ structured PSCs with a decent open-circuit voltage of $1.15 \mathrm{~V}$ and a fill factor of 0.81. This all-around nanoscale grain wrapping leads to remarkable improvement of the operational stability of PSCs, sustaining $80 \%$ of the efficiency after "burn-in" under full sunlight with UV for more than $5200 \mathrm{~h}$. Our findings provide a new pathway towards efficient and stable PSCs.

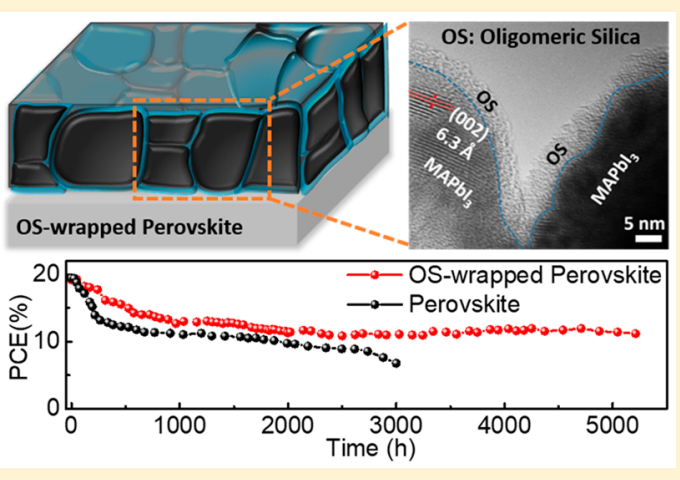

$\mathrm{T}$ he emerged organic-inorganic halide perovskites are currently the focus of the photovoltaic research community, and the certified power conversion efficiency (PCE) has reached $24.2 \%$ owing to their intriguing optoelectronic properties. ${ }^{1-5}$ However, the long-term stability of perovskite solar cells (PSCs) remains the bottleneck hampering the progress toward future commercialization. The degradation of PSCs is reported to be accelerated by external stimuli such as moisture, ${ }^{6,7}$ ultraviolet light, ${ }^{8,9}$ oxygen, ${ }^{8,10}$ and heat. ${ }^{11,12}$ The corrosion of charge transport layers (CTLs) and metal contacts that originated from ion migration was recognized as another significant degradation channel in PSCs. Various strategies including interface modification, ${ }^{13-16}$ CTL engineering, ${ }^{17-19}$ composition substitution, $^{3,20,21}$ and corrosion-blocking layer engineering ${ }^{18,22}$ have been shown to enhance the stability of PSCs in ambient environment. These approaches indeed extended the lifespan of PSCs, but a substantial gap in terms of long-term operational stability still exists between PSCs and commercial photovoltaics. This indicates that there remain intrinsic factors that will drive the degradation with aging. The study of an intrinsic instability mechanism in PSCs has gained increasing

Received: March 20, 2019

Accepted: May 7, 2019

Published: May 7, 2019 


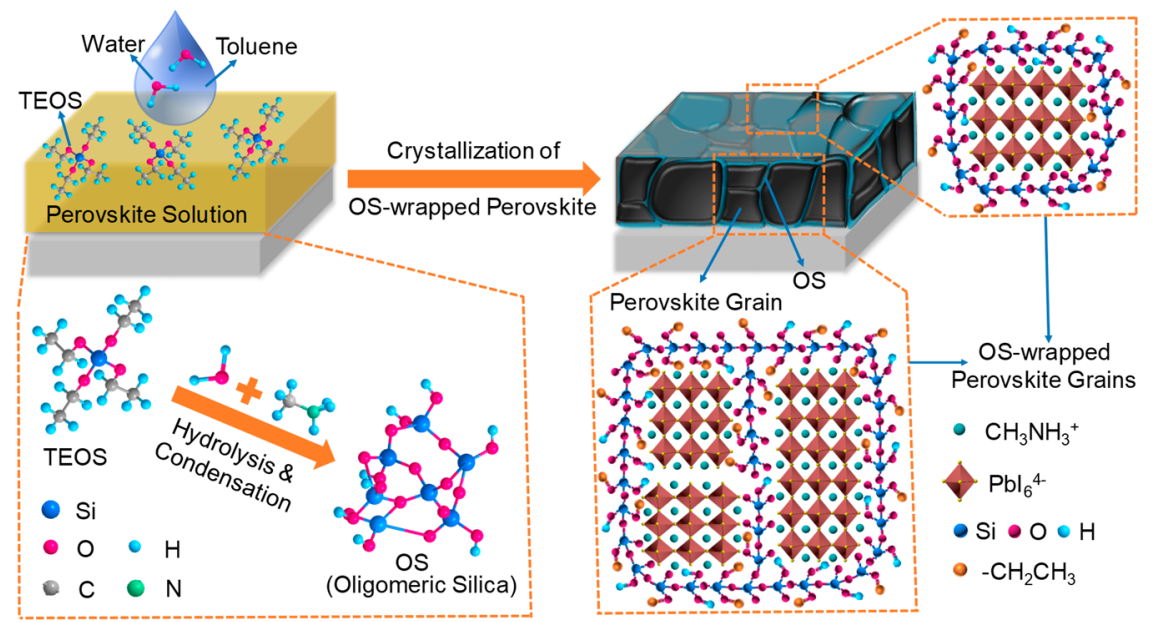

Figure 1. Schematic illustration showing the in situ formation process of an OS-wrapped perovskite thin film and the hypothesized perovskite-OS nanostructures.

attention in recent years. It is widely recognized that the high density of ionic defects at the surfaces and grain boundaries (GBs) of perovskite films would affect the open-circuit voltage $\left(V_{\mathrm{OC}}\right)$ of the devices due to the nonradiative recombination. ${ }^{23,24}$ Recently, these defect states were also found to be detrimental to the long-term stability of PSCs. ${ }^{25,26}$ Choi et al. revealed that the irreversible dissociation of perovskites in the presence of moisture is triggered by the charges trapped along GBs, which assist in deprotonation of organic cations by an induced local electric field. ${ }^{27}$ Our previous study of moisturedependent perovskite grain stability also clearly showed that the decomposition of perovskite grains was initialized by the defective surface and GBs. ${ }^{7}$ In addition, from the device point of view, Song et al. demonstrated that the trap states derived from the increased defects at the surface and GBs of perovskites accelerated the performance deterioration in PSCs. $^{28}$ Even for encapsulated PSCs, it is crucial to control the defects in perovskite materials and at the interfaces, which are believed to be the cause of $V_{\mathrm{OC}}$ loss and performance degradation with aging. ${ }^{29}$ Moreover, theoretical studies suggested that the ion migration and formation of defects including vacancies $\left(\mathrm{V}_{\mathrm{MA}}, \mathrm{V}_{\mathrm{Pb}}, \mathrm{V}_{\mathrm{I}}\right)$, antisites $\left(\mathrm{MA}_{\mathrm{Pb}}, \mathrm{Pb}_{\mathrm{MA}}\right.$ $\left.\mathrm{MA}_{\mathrm{I}}, \mathrm{Pb}_{\mathrm{I}}, \mathrm{I}_{\mathrm{Pb}}\right)$, and interstitials $\left(\mathrm{MA}_{\mathrm{i}}, \mathrm{Pb}_{\mathrm{i}}, \mathrm{X}_{\mathrm{i}}\right)$ would not only deteriorate the optoelectronic properties of perovskite layer but also induce interface degradation leading to dominant effects on device performance and stability. ${ }^{30,31}$ The passivation of the trap states at the surface and GBs of perovskite films is critically important in addressing the intrinsic instability of PSCs. The reported strategies employing fullerene, ${ }^{32}$ pyridine, ${ }^{33}$ zwitterion molecules, ${ }^{25}$ and polymers ${ }^{34}$ for film surface passivation all effectively reduced the charge trap density and elongated the carrier recombination lifetime, leading to improved device efficiency. However, these approaches provide limited protection to perovskite films from external stimuli. Therefore, alternative approaches that aim at synchronously passivating and stabilizing perovskite grains are imperative to boost their photovoltaic performance and further prolong the operational lifespan of PSCs.

Here, we report a facile strategy of in situ wrapping perovskite grains within an oligomeric silica (OS) matrix by finely controlling the hydrolysis and condensation of tetraethyl orthosilicate (TEOS) in the presence of limited water during perovskite crystallization (Patent filed in 2016). For the modified perovskite thin film with an OS layer, we observe a remarkably reduced trap density and prolonged carrier recombination lifetime, which boosts the $V_{\mathrm{OC}}$ up to $1.16 \mathrm{~V}$ for $\mathrm{p}-\mathrm{i}-\mathrm{n}$ planar $\mathrm{CH}_{3} \mathrm{NH}_{3} \mathrm{PbI}_{3}\left(\mathrm{MAPbI}_{3}\right)$ PSCs with a champion PCE of $21.1 \%$. We propose that the improved performance is due to the passivation of undercoordinated $\mathrm{Pb}$ defects via a coordination bond between the oxygen atom (a Lewis base site) of the $-\mathrm{OCH}_{2} \mathrm{CH}_{3}$ group in the OS layer with undercoordinated lead ions at the perovskite surface and GBs. The universal passivation effect of OS shells for perovskite films with different compositions enables a further improved PCE of $21.5 \%$ for the PSCs with $\mathrm{FA}_{0.85} \mathrm{MA}_{0.15} \mathrm{~Pb}\left(\mathrm{I}_{0.85} \mathrm{Br}_{0.15}\right)_{3}$ films. Moreover, the OS shell acting as a physical barrier not only prevents the permeation of external moisture and atmospheric gases but also may suppress the internal ion migration and perovskite decomposition by blocking both internal and external degradation channels in perovskite films. Benefiting from this all-around grain stabilization at the nanoscale, the corresponding PSCs show both greatly enhanced moisture resistance and operational stability.

Due to its anomalously high stability, ease of growth, and optical transparency, silica coating over colloidal particles, polymers, and surfactant aggregates has been extensively investigated in materials science and chemistry for enhancement of colloidal stability. ${ }^{35}$ The Stöber method, ${ }^{36}$ which involves hydrolysis and polycondensation of silicon alkoxides (e.g., TEOS) in the presence of ammonia as a catalyst and alcohol-water media, is one of the most widely explored approaches for synthesizing nanoparticles with core-shell (silica as shell) geometries. ${ }^{37-39}$ To realize all-around wrapping of perovskite grains within silica shells that have great potential in mitigating the defect-driven degradation of perovskite thin films, we rationally integrated the classical Stöber reaction with the solution-processed perovskite crystallization. Previous studies that explore the protection of perovskite nanocrystals or large grains usually employ pure silica as the coating material; ${ }^{39,40}$ however, $\mathrm{SiO}_{2}$ is a Lewis acid, which has little interaction with positively charged ions in perovskites such as $\mathrm{Pb}^{2+}$. In addition, silica of high polymerization degree will constrain the perovskite growth significantly. ${ }^{40}$ We propose to control the hydrolysis and condensation of TEOS by introducing a limited amount of water to the system, leading to the formation of OS with an 

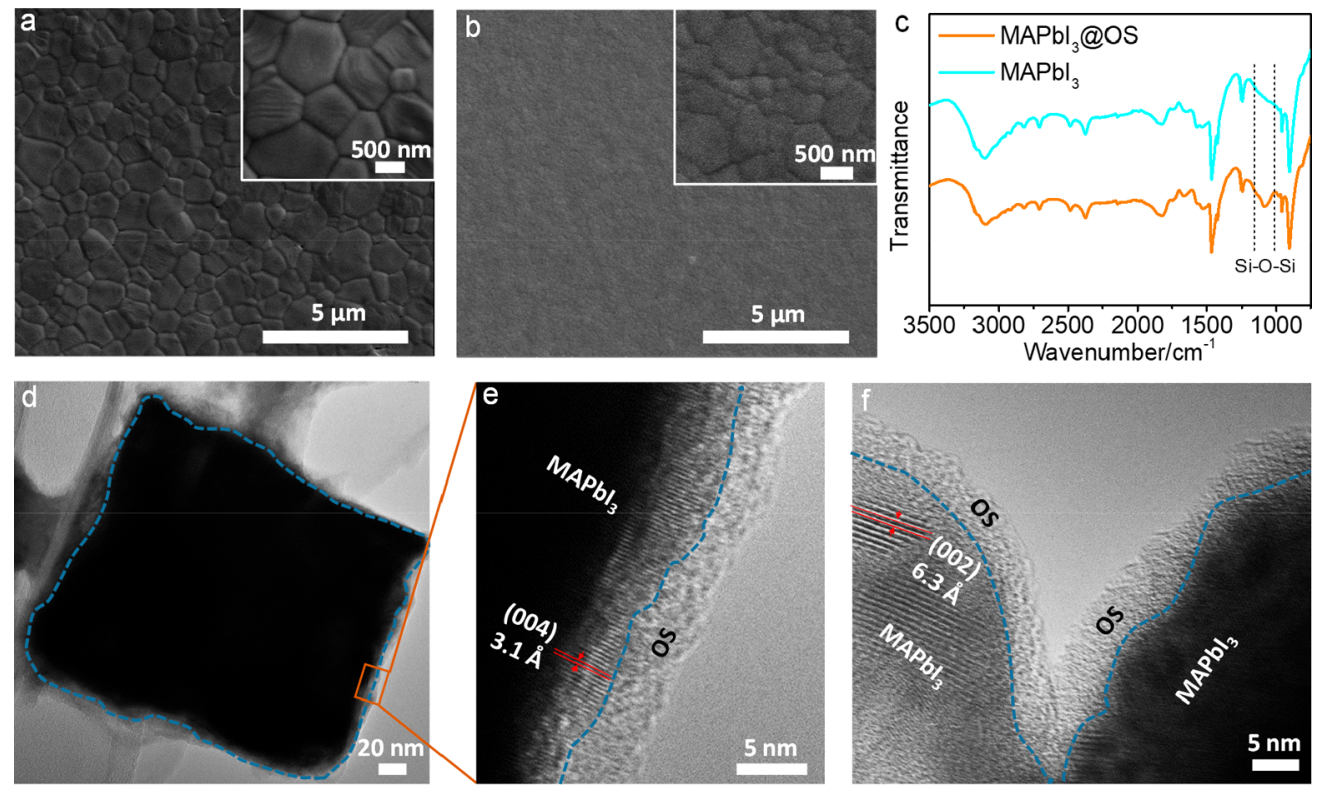

Figure 2. (a,b) Top-view SEM images and (c) FTIR spectra of $\mathrm{MAPbI}_{3}$ and $\mathrm{MAPbI}_{3} @ O \mathrm{OS}$ films; (d) TEM image of a single perovskite grain wrapped within a silica matrix; (e) high-resolution TEM image of the boundary region (rectangle area); (f) high-resolution TEM image of the junction region of two adjacent perovskite grains.

$-\mathrm{OCH}_{2} \mathrm{CH}_{3}$ group containing electron-rich Lewis base sites. Figure 1 illustrates the in situ formation of an OS-wrapped perovskite thin film via an antisolvent-assisted crystallization approach. TEOS was used as the silica source and directly introduced into the perovskite $\left(\mathrm{MAPbI}_{3}\right)$ precursor solution with various volume ratios $(3,5,10$ vol \%). The methylamine in this mixed solution served as the role of catalyst as that of ammonia in a typical Stöber reaction. The small amount of water in the antisolvent, toluene (we use reagent grade instead of anhydrous solvent), initialized the hydrolysis of TEOS monomers, which formed hydroxides with liberation of ethyl alcohol. The hydrolyzed silicates then condensed to form polymeric silica that was accelerated by the amine catalyst. The hydrolysis and polycondensation of TEOS occurred synchronously with perovskite crystallization, allowing in situ formation of an OS matrix over perovskite grains. We hypothesize that an OS matrix wrapping a single grain or multiple perovskite grains prefers to link with excess ions such as $\mathrm{Pb}^{2+}$ and methylammonium ions $\left(\mathrm{MA}^{+}\right)$via electrostatic interaction at surfaces and GBs, as depicted in Figure 1.

To study the effect of OS coating on the perovskite crystal structure, we carried out X-ray diffraction (XRD) analysis on the as-prepared perovskite thin films. As shown in Supplementary Figure 1, all of the thin films exhibit similar XRD patterns, and the featured diffraction peaks can be indexed to the typical $\mathrm{MAPbI}_{3}$ perovskite, which confirms the high phase purity and well-preserved crystallinity. No diffraction peak was observed for the OS within the thin films, which suggests that the OS derived from the TEOS hydrolysis and condensation is amorphous. With increasing the concentration of TEOS, the full width at half-maximum (fwhm) of the main peak at $14^{\circ}$ slightly increased from 0.163 to 0.206 , indicating the formation of smaller perovskite crystals. Top-view scanning electron microscopy (SEM) was then employed to investigate the morphology change of perovskite films after introducing TEOS in the precursor solution. Unless stated otherwise, all of the following characterizations were based on the perovskite films that were derived from the perovskite precursor solution containing 5 vol \% TEOS. The pristine perovskite film shown in Figure $2 \mathrm{a}$ is compact and pinhole-free, with an average grain size of $\sim 1 \mu \mathrm{m}$. After the incorporation of TEOS, the GBs were obscure in the as-prepared perovskite film (Figure 2b), which indicated that the surface and GBs of perovskite grains might have been fully coated with an OS layer. A slight reduction in grain size was observed in the film shown in the inset of Figure $2 \mathrm{~b}$, which is consistent with XRD results. During the perovskite crystallization, the interpenetrated silica growth may truncate the perovskite crystals and/or prevent their further growth, thereby shrinking the perovskite grain size. To verify the formation of OS coating over perovskite grains, we performed Fourier transform infrared spectroscopy (FTIR) analysis on the perovskite films fabricated without and with TEOS. As shown in Figure 2c, a new and wide vibrational peak between 1160 and $1011 \mathrm{~cm}^{-1}$ appeared in the corresponding FTIR spectrum after introducing TEOS, which can be ascribed to the $\mathrm{Si}-\mathrm{O}-\mathrm{Si}$ bonds ${ }^{19}$ and thus confirms the formation of OS within the perovskite film. To elucidate the detailed microstructure as well as the distribution of OS in the perovskite film, transmission electron microscopy (TEM) analysis was carried out (Figure $2 \mathrm{~d}, \mathrm{e}$ ). To prepare the TEM sample, we scraped the perovskite thin film off from the substrate and dispersed it in anhydrous toluene with ultrasonication treatment for $1 \mathrm{~min}$. The as-prepared dispersion was directly dropped on the mesh copper TEM grid. Figure $2 \mathrm{~d}$ is the TEM image of a single perovskite grain, which shows significant contrast difference between the center (dark) and boundary (light). To further investigate the detailed microstructure, a high-resolution TEM image (Figure 2e) was then acquired at the boundary region (rectangle area), which clearly exhibits the contrast difference between the core and the shell. The interplanar spacing of $\sim 3.1 \AA$ of the core corresponds to the (004) plane of $\mathrm{MAPbI}_{3}$ perovskite, which is consistent with the XRD result. We also investigated the junction region of two adjacent perovskite grains, as shown in Figure $2 \mathrm{f}$. The lattice fringes in the grain core can be indexed to the (002) planes of $\mathrm{MAPbI}_{3}$ with an interplanar spacing of 

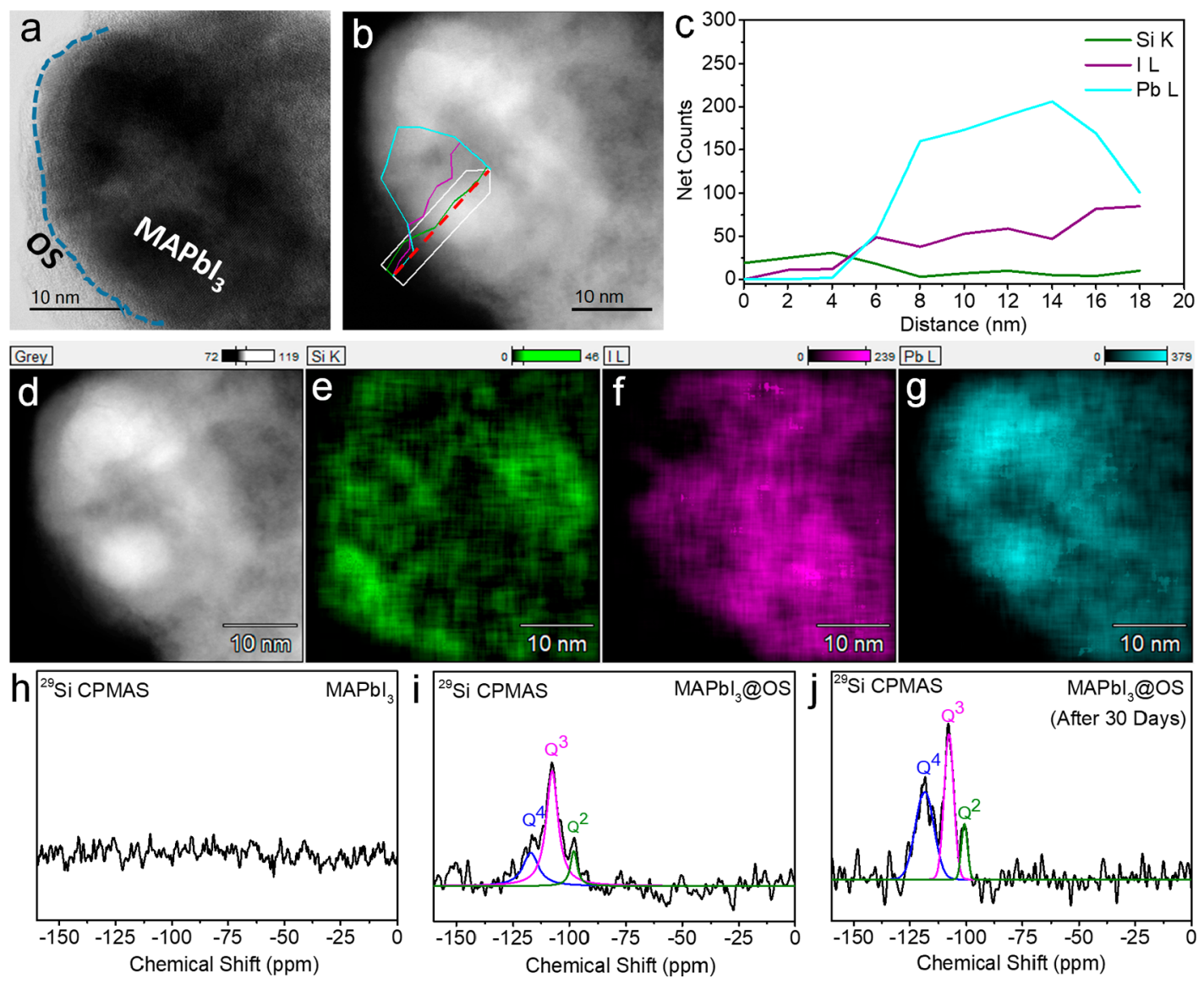

Figure 3. (a) Bright-field STEM image of the typical MAPbI ${ }_{3} @ O S$ nanostructure; (b) corresponding dark-field STEM image including a red dashed line indicating the location of the scan; the green, purple, and cyan lines represent $\mathrm{Si}$, I, and Pb elements, respectively; (c) STEMEDS line scan profiles of $\mathrm{Si}$, I, and Pb elements; (d) dark-field STEM image of the same $\mathrm{MAPbI}_{3} @ O$ OS nanostructure; STEM-EDS elemental maps for Si (e), Pb (f), and I (g); solid-state ${ }^{29} \mathrm{Si}$ MAS NMR spectra of (h) pristine $\mathrm{MAPbI}_{3},(\mathrm{i})$ fresh $\mathrm{MAPbI}_{3} @ O \mathrm{OS}$, and (j) MAPbI ${ }_{3} @ O S$ after exposure to ambient air for 30 days.

$6.3 \AA$ A. We observe that the continuous OS layer not only covers on the surfaces of these two grains but also penetrates into the GB. To further define the distribution of OS in the $\mathrm{MAPbI}_{3} @ \mathrm{OS}$ core-shell nanostructure, we conducted scanning TEM (STEM)-energy dispersive X-ray spectroscopy (EDS) analysis on this composite sample. Figure $3 \mathrm{a}$ is a bright-field STEM image of the typical $\mathrm{MAPbI}_{3} @ \mathrm{OS}$ featuring an obvious core-shell nanostructure, and the corresponding dark-field STEM image is shown in Figure $3 b$, including a red dashed line indicating the location of the EDS line scan across the outer thin layer (shell) to the inner perovskite grain (core). The corresponding EDS line profile is shown in Figure 3c. In the shell region, $\mathrm{Si}$ was identified as the dominant element and no obvious $\mathrm{Pb}$ and I signal was observed, which indicates that the outer amorphous thin layer is mainly composed of Si. When the line scan comes to the perovskite grain, all of the elements including $\mathrm{Pb}, \mathrm{I}$, and $\mathrm{Si}$ are present in the core region. To further investigate their distribution in the whole nanostructure, we performed EDS elemental mapping. Figure $3 \mathrm{~d}-\mathrm{f}$ corresponds to the elemental maps of $\mathrm{Si}, \mathrm{Pb}$, and I, and we found that all of the elements are homogeneously distributed in the inner core region, while the outer shell has only Si element observed. We also carried out EDS elemental mapping analysis on the composite sample on a large scale, and the distribution of $\mathrm{Si}$ was found to be as uniform as $\mathrm{Pb}$ and I elements, as shown in EDS elemental maps (Supplementary
Figure 2). To determine the amount of silica within the OSwrapped perovskite thin film (with 5 vol \% TEOS), three spots in the composite sample were randomly selected to acquire EDS spectra (Supplementary Figure 3), and the atomic ratio of $\mathrm{Si}$ in the film was approximately $1.2-1.3 \%$. All of these characterization results have provided solid evidence verifying our hypothesis that the perovskite grains were successfully wrapped inside of the OS matrix and the nanoscale coating covered the surfaces and GBs in the thin films.

To gain insight into the composition of the OS layer in the $\mathrm{MAPbI}_{3} @ \mathrm{OS}$ thin films, solid-state nuclear magnetic resonance (ss-NMR) was utilized. For ss-NMR measurements, we scraped the perovskite thin films off from the substrates and collected ca. $50 \mathrm{mg}$ of powder for each sample. The ${ }^{13} \mathrm{C} \mathrm{CP} /$ MAS ss-NMR spectra of the pristine $\mathrm{MAPbI}_{3}$ and $\mathrm{MAPbI}_{3} @$ OS samples are presented in Supplementary Figure 4. Both samples showed a signal at $30 \mathrm{ppm}$ assigned to the carbon species of $\mathrm{CH}_{3} \mathrm{NH}_{3} \mathrm{PbI}_{3}$. ${ }^{41}$ An additional peak at $42 \mathrm{ppm}$ was observed in the ${ }^{13} \mathrm{C} \mathrm{CP} / \mathrm{MAS}$ ss-NMR spectrum of the $\mathrm{MAPbI}_{3} @ \mathrm{OS}$ sample, which is attributed to the ${ }^{1} \mathrm{C}$ carbon species of $\mathrm{Si}-\mathrm{O}-{ }^{1} \mathrm{CH}_{2}{ }^{2} \mathrm{CH}_{3}$. This verified the presence of $-\mathrm{CH}_{2} \mathrm{CH}_{3}$ in the OS layer due to the incomplete hydrolysis and condensation of TEOS. We speculate that the signal of ${ }^{2} \mathrm{C}$ carbon species from $\mathrm{Si}-\mathrm{O}-{ }^{1} \mathrm{CH}_{2}{ }^{2} \mathrm{CH}_{3}$ in the OS layer was superimposed with the peak of methyl carbon species in $\mathrm{CH}_{3} \mathrm{NH}_{3} \mathrm{PbI}_{3}$. ${ }^{29} \mathrm{Si} \mathrm{CP} / \mathrm{MAS}$ ss-NMR is useful to monitor the 

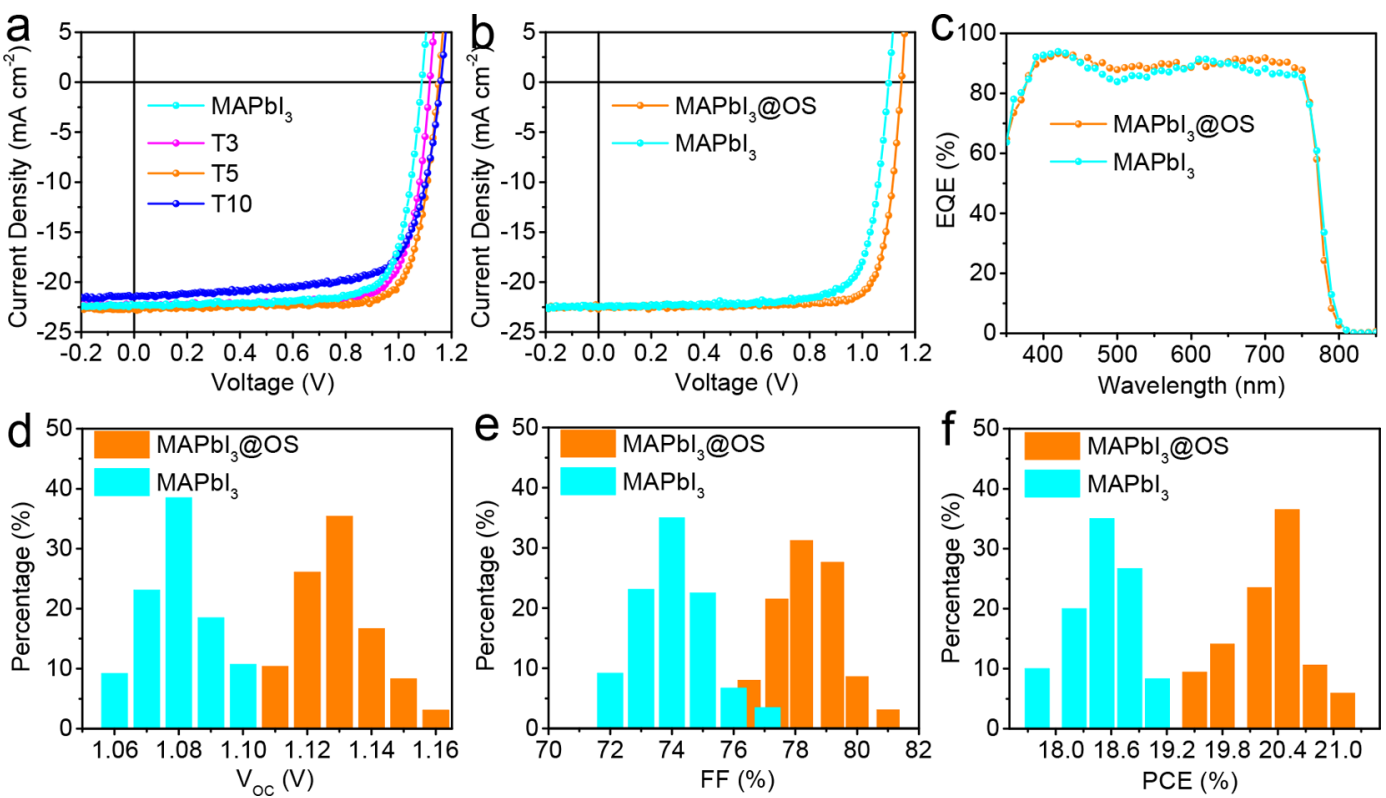

Figure 4. (a) Photocurrent density-voltage $(J-V)$ curves of PSCs based on MAPbI ${ }_{3}$ and varying MAPbI ${ }_{3} @ O S$ films (T3:3 vol \% TEOS, T5:5 vol \% TEOS, T10:10 vol \%); (b) $J-V$ curves and (c) EQE of the optimized devices; statistics of the $V_{\mathrm{OC}}(\mathrm{d})$ ), FF (e), and PCE (f) distribution for devices with $\mathrm{MAPbI}_{3}$ (20 devices) and $\mathrm{MAPbI}_{3} @ O S$ (36 devices, 5 vol \% TEOS) films.

degree of TEOS hydrolysis and condensation and to differentiate the composition of the resultant OS layer. Figure $3 \mathrm{~g}-\mathrm{i}$ exhibits the ${ }^{29} \mathrm{Si} \mathrm{CP} / \mathrm{MAS}$ ss-NMR spectra for the pristine $\mathrm{MAPbI}_{3}$ sample and $\mathrm{MAPbI}_{3} @ \mathrm{OS}$ samples before and after exposure in ambient air. No Si signal was observed in the spectrum (Figure $3 \mathrm{~g}$ ) of the pristine $\mathrm{MAPbI}_{3}$ sample. The spectrum (Figure 3h) of the as-prepared $\mathrm{MAPbI}_{3} @ \mathrm{OS}$ showed three signals at $-97,-107$, and $-117 \mathrm{ppm}$, corresponding to $\mathrm{Q}^{2}\left(\mathrm{Si}(\mathrm{OSi})_{2}(\mathrm{OX})_{2}, \mathrm{X}=\mathrm{H}\right.$ or $\left.\mathrm{CH}_{2} \mathrm{CH}_{3}\right), \mathrm{Q}^{3}\left(\mathrm{Si}(\mathrm{OSi})_{3}(\mathrm{OX})\right)$, and $\mathrm{Q}^{4}\left(\mathrm{Si}(\mathrm{OSi})_{4}\right)$ species, which further verified the formation of OS during perovskite crystallization due to the incomplete hydrolysis and condensation of TEOS. Obviously, the ratio of $\mathrm{Q}^{4}\left(\mathrm{Q}^{4} / \mathrm{Q}^{2}+\mathrm{Q}^{3}+\mathrm{Q}^{4}\right)$ in the $\mathrm{MAPbI}_{3} @ \mathrm{OS}$ increased after exposure to ambient air for 1 month, which suggested that the condensation degree of the OS layer was increased. This may further extend the stability of the $\mathrm{MAPbI}_{3} @ \mathrm{OS}$ in a humid environment. We also performed elemental microanalysis on the as-prepared $\mathrm{MAPbI}_{3} @ \mathrm{OS}$ sample (5 vol \% TEOS in the precursor solution) to quantify the loss of $\mathrm{CH}_{2} \mathrm{CH}_{3}$ from TEOS, which helped understand the degree of TEOS hydrolysis and condensation. The element $(\mathrm{N}, \mathrm{C}$, and $\mathrm{H}$ ) weight ratios are summarized in the Supplementary Table 1. On the basis of the calculation, we determine that ca. $53.9 \%$ of $\mathrm{CH}_{2} \mathrm{CH}_{3}$ from TEOS was lost in the fabrication of the $\mathrm{MAPbI}_{3} @ \mathrm{OS}$ film.

To investigate the impact of OS coating on photovoltaic performance, we fabricated $\mathrm{p}-\mathrm{i}-\mathrm{n}$ planar PSCs, which have a device structure as shown in the scheme (Supplementary Figure 5) ${ }^{19,42}$ Because the thickness of the OS layer is critically important in determining the charge transport property and thereby photovoltaic performance, we first tried to find out the best concentration of TEOS additive. Figure $4 \mathrm{a}$ shows the typical photocurrent density-voltage $(J-V)$ curves of the PSCs based on the pristine perovskite film and varying OSwrapped perovskite films fabricated with different amounts of TEOS additives. The control device without OS had a shortcircuit current density $\left(J_{\mathrm{SC}}\right)$ of $22.3 \mathrm{~mA} \mathrm{~cm}{ }^{-2}$, an open-circuit voltage $\left(V_{\mathrm{OC}}\right)$ of $1.09 \mathrm{~V}$, and a fill factor (FF) of $76.5 \%$, yielding a moderate PCE of $18.6 \%$, which is consistent with our previous reported work. ${ }^{19}$ When 3 vol \% TEOS was blended into the precursor solution, the $V_{\mathrm{OC}}$ and FF increased to $1.12 \mathrm{~V}$ and $77.4 \%$, respectively, and the corresponding device T3 delivered a higher PCE of $19.3 \%$. By adding 5 vol \% TEOS, the PCE of device T5 was boosted to $20.6 \%$ with a large $V_{\mathrm{OC}}$ of $1.15 \mathrm{~V}$. No obvious photocurrent hysteresis was observed in the device made with $\mathrm{MAPbI}_{3} @ \mathrm{OS}$ (5 vol \% TEOS) by changing the sweeping direction (Supplementary Figure 6). Further increasing the ratio of TEOS to $10 \mathrm{vol} \%$ deteriorated the device (T10) performance with lower $J_{S C}$ and $\mathrm{FF}$, though $V_{\mathrm{OC}}$ was increased to $1.16 \mathrm{~V}$. The reduced efficiency can be attributed to the poorer charge collection induced by thicker OS shells. All of the average photovoltaic parameters of these corresponding perovskite devices are summarized in Supplementary Table 2 . Figure 4 b shows the $J-$ $V$ curves of the champion devices based on pristine and OSwrapped (with $5 \mathrm{vol} \%$ TEOS) $\mathrm{MAPbI}_{3}$ perovskite films. The best control device without OS coating delivered a PCE of $19.1 \%$, as shown in Table 1 . After optimal OS coating, the champion OS-wrapped perovskite device exhibited a $J_{\mathrm{SC}}$ of $22.7 \mathrm{~mA} \mathrm{~cm}^{-2}$, a $V_{\mathrm{OC}}$ of $1.15 \mathrm{~V}$, and a $\mathrm{FF}$ of $80.9 \%$, yielding a decent PCE of $21.1 \%$, which is among the top efficiencies reported for $\mathrm{MAPbI}_{3}$-based solar cells (Supplementary Table 3 ). The integrated $J_{S C}$ from external quantum efficiency (EQE) spectra shown in Figure $4 \mathrm{c}$ reached 22.0 and $22.3 \mathrm{~mA} \mathrm{~cm}^{-2}$ for

Table 1. Photovoltaic Performance Parameters of PSCs Fabricated with Pristine and Silica-Wrapped Perovskite Films

\begin{tabular}{lcccc}
\multicolumn{1}{c}{ sample } & $\begin{array}{c}J_{s c} \\
\left(\mathrm{~mA} \mathrm{~cm}^{-2}\right)\end{array}$ & $V_{\mathrm{OC}}(\mathrm{V})$ & $\mathrm{FF}(\%)$ & $\eta(\%)$ \\
$\mathrm{MAPbI}_{3}$ & 22.5 & 1.10 & 77.1 & 19.1 \\
$\mathrm{MAPbI}_{3} @ \mathrm{OS}$ & 22.7 & 1.15 & 80.9 & 21.1 \\
$\mathrm{FA}_{0.85} \mathrm{MA}_{0.15} \mathrm{~Pb}\left(\mathrm{I}_{0.85} \mathrm{Br}_{0.15}\right)_{3}$ & 23.2 & 1.10 & 77.6 & 19.8 \\
$\mathrm{FA}_{0.85} \mathrm{MA}_{0.15} \mathrm{~Pb}\left(\mathrm{I}_{0.85} \mathrm{Br}_{0.15}\right)_{3} @$ & 23.1 & 1.15 & 81.1 & 21.5 \\
$\quad \mathrm{OS}$ & & & &
\end{tabular}



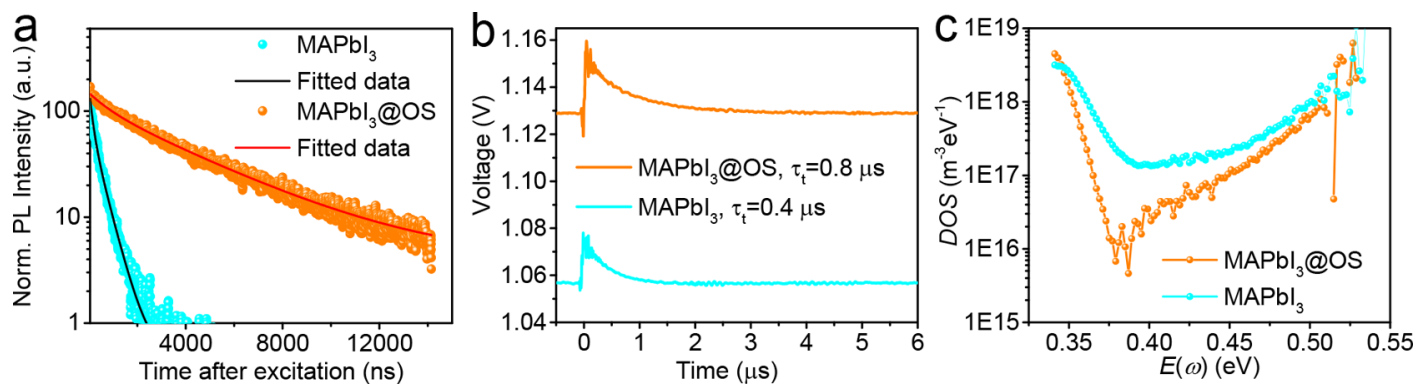

Figure 5. (a) Time-resolved PL decay comparison for $\mathrm{MAPbI}_{3}$ and $\mathrm{MAPbI}_{3} @ O \mathrm{OS}$ films, (b) TPV decay curves, and (c) tDOS obtained for perovskite devices based on $\mathrm{MAPbI}_{3}$ and $\mathrm{MAPbI}_{3} @ O \mathrm{OS}$ films.

the control and silica-wrapped perovskite devices, respectively, which are in good agreement with those from $J-V$ measurements. It is noted that PCE enhancement mainly comes from the improved $V_{\mathrm{OC}}$ and $\mathrm{FF}$, which we assume are due to the significantly reduced trap states and thereby suppressed nonradiative recombination in OS-wrapped perovskite devices. The statistics of $V_{\mathrm{OC}}, \mathrm{FF}$, and PCE distributions shown in Figure $4 \mathrm{~d}-\mathrm{f}$ demonstrated the reliability and repeatability of the performance enhancement obtained by employing OSwrapped perovskite.

Early studies have shown that the loss of halide and MA during the annealing process of $\mathrm{MAPbI}_{3}$ perovskites may result in undercoordinated $\mathrm{Pb}$ atoms both on the crystal surface and at the GBs. ${ }^{33}$ According to recent theoretical modeling and experimental studies, it is widely accepted that these undercoordinated $\mathrm{Pb}$ atoms, or/and $\mathrm{Pb}$ clusters in some cases, can act as electronic trap states within the perovskite material. The undercoordination of the $\mathrm{Pb}$ atoms could induce the formation of net positive charges on the atom, which would then facilitate the coordination or bond formation with electron-rich Lewis bases. Recently, several Lewis base materials have been employed to effectively passivate the defects at the surface and GBs of perovskite films. ${ }^{33,43,44}$ As discussed earlier, the solid-state $\mathrm{NMR}$ spectra data has confirmed the presence of an $-\mathrm{OCH}_{2} \mathrm{CH}_{3}$ group in the OS layer due to the controlled degree of TEOS hydrolysis and condensation. The oxygen atom in the $-\mathrm{OCH}_{2} \mathrm{CH}_{3}$ group is a Lewis base (electron donor) site due to the electron pair associated with the $\mathrm{C}-\mathrm{O}$ bond, ${ }^{44}$ while pure $\mathrm{SiO}_{2}$ is a Lewis acid. The electrostatic attraction between the local negative charge surrounding the oxygen atoms and the positive charge on the undercoordinated $\mathrm{Pb}$ could result in the formation of a coordinate or dative covalent bond. Those positive charges can then be delocalized within the OS layer. Thus, we suppose that the nanoscale OS shell may also be able to reduce the density of defects at the surfaces and GBs of perovskites effectively.

In order to verify our hypothesis and reveal the mechanisms for the performance enhancement induced by the OS coating, we first conducted steady-state photoluminescence (PL) and time-resolved PL (TRPL) decay measurements on perovskite thin films with and without OS coating on glass substrates. The PL intensity of perovskite with OS coating is over 3 times higher than that of the pristine one (Supplementary Figure 7), indicating that the OS layer could passivate the defects in the perovskite film. As shown in Figure 5a, the TRPL decay of perovskite films with and without OS coating showed a biexponential decay with a fast and a slow component. All of the fitted TRPL parameters for both samples are summarized in Supplementary Table 4. For the slow component, an extremely long carrier lifetime of $3.9 \mu$ s was deduced from the fitted TRPL decay curve of the $\mathrm{MAPbI}_{3} @ \mathrm{OS}$ film, which was substantially longer than that $(482 \mathrm{~ns})$ of the pristine $\mathrm{MAPbI}_{3}$ film. The prolonged charge recombination lifetime indicates suppressed charge recombination at the surface and/or GBs, which can be attributed to the trap-state passivation of the OS layer, agreeing with our proposed mechanism. To find out how passivation affects the charge recombination process in the operating devices, transient photovoltage (TPV) decay measurements were conducted on the perovskite devices with and without OS coating. Both devices were soaked under 1 sun illumination, and laser pulses ( $337 \mathrm{~nm}, 4 \mathrm{~ns}$ ) were applied to disturb the open-circuited devices to trigger a small transient photovoltaic signal. ${ }^{25}$ As seen in Figure $5 b$, the charge recombination lifetime of the $\mathrm{MAPbI}_{3} @ \mathrm{OS}$ device was doubled to $0.8 \mu \mathrm{s}$ in comparison with that $(0.4 \mu \mathrm{s})$ of the control device. The TPV result also indicates that the perovskite device with OS passivation showed a larger average photovoltage of $1.13 \mathrm{~V}$ than the devices without OS $(1.06 \mathrm{~V})$, which is in accordance with the $J-V$ measurement.

The reduction of trap states in $\mathrm{MAPbI}_{3}$ films by OS passivation was quantified by using the thermal admittance spectroscopy (TAS) method. TAS is a well-established technique for characterizing both shallow and deep defects in various types of solar cells. ${ }^{32,45,46}$ The energy profile of the trap density of states (tDOS) can be derived using the method described in the Supporting Information. As shown in Figure $5 c$, the device with OS-wrapped perovskite exhibited significantly lower tDOS over the whole trap depth region compared to the control device. In the deeper trap region $(0.40-0.52 \mathrm{eV})$, which was assigned to defects at the film surface, $^{25,32}$ the density of defect states of $1 \times 10^{16}-1 \times 10^{17}$ $\mathrm{m}^{-3}$ in the $\mathrm{MAPbI}_{3} @ \mathrm{OS}$ device is $2-4$ times smaller than that of the devices with pristine $\mathrm{MAPbI}_{3}$. In addition, the density of shallower trap states $(0.35-0.40 \mathrm{eV})$, which was assigned to traps at $\mathrm{GBs}^{32}{ }^{32}$ decreased by up to 1 order of magnitude after OS coating in the $\mathrm{MAPbI}_{3} @ O$ OS devices. This indicates that silica not only reduced the tDOS at surfaces but also effectively passivated the trap states located at GBs, leading to suppressed trap-assisted nonradiative recombination and thus longer carrier lifetime, as revealed above.

Theoretically, the $V_{\mathrm{OC}}$ upper limit in PSCs is determined by the quasi-Fermi level splitting of electrons $\left(E_{\mathrm{fn}}\right)$ and holes $\left(E_{\mathrm{fp}}\right)$ in the perovskite layer under illumination, which is likely to be pinned at the interfaces or GBs by the trap states. ${ }^{47}$ As suggested by the TAS analysis, the pristine $\mathrm{MAPbI}_{3}$ film had a relatively larger tDOS, which resulted in reduced quasi-Fermi level splitting and a smaller $V_{\mathrm{OC}}$. With OS coating, the device fabricated with the $\mathrm{MAPbI}_{3} @ \mathrm{OS}$ film showed a significantly 

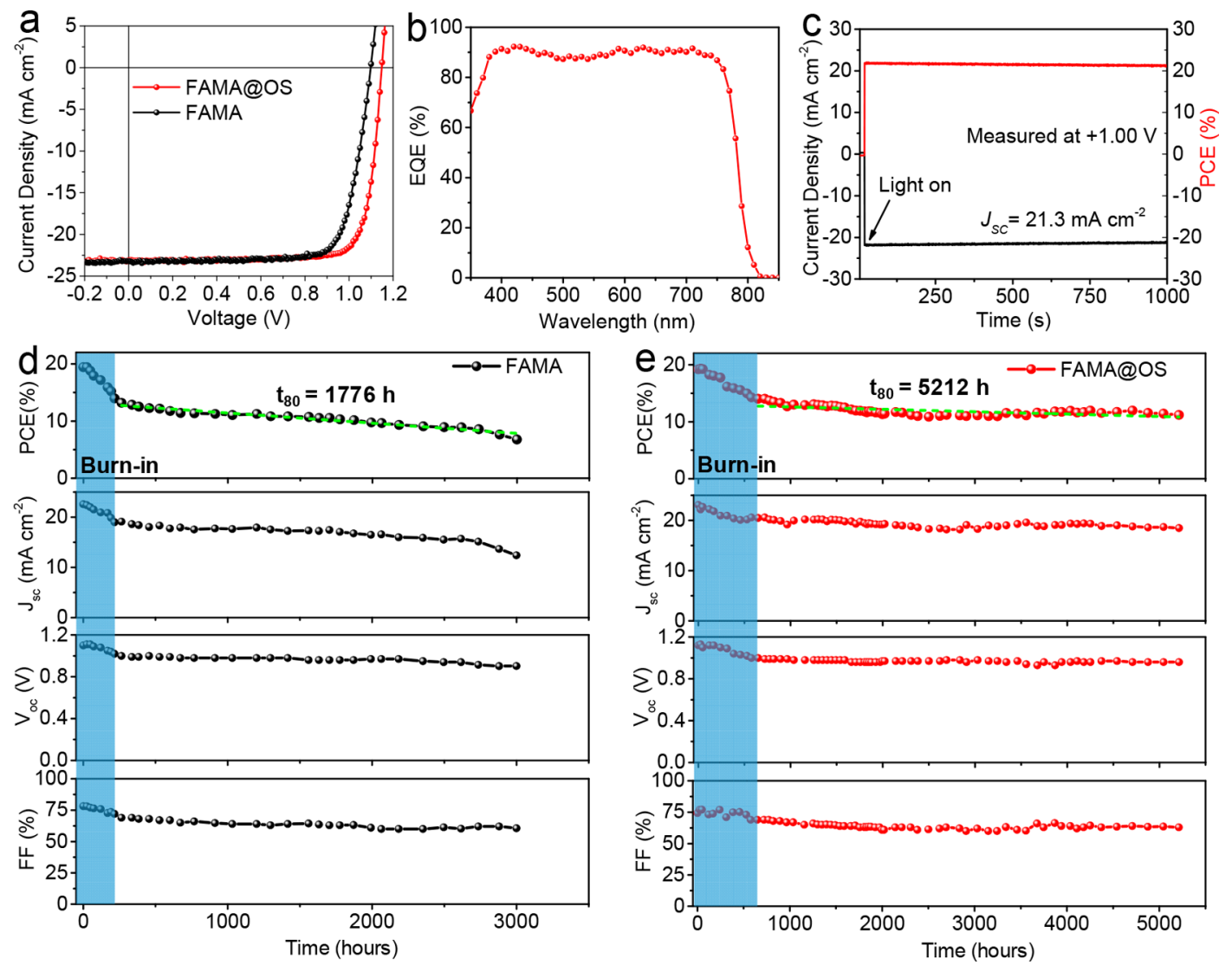

Figure 6. (a) $J-V$ curves of optimized PSCs based on FAMA and FAMA@OS perovskite films; (b) EQE and (c) steady-state photocurrent and efficiency of the champion PSCs fabricated with FAMA@OS perovskite films; evolution of photovoltaic performance parameters of the typical encapsulated devices with FAMA (d) and FAMA@OS (e) perovskite films as a function of illumination time.

higher $V_{\mathrm{OC}}$, which we assume can be attributed to the mitigated pinning of quasi-Fermi level splitting benefiting from the reduction of trap states by OS passivation. To confirm whether the increased quasi-Fermi level splitting is from the upward shift of $E_{\mathrm{fn}}$ or downward shift of $E_{\mathrm{fp}}$, we conducted additional experiments. Various amounts of TEOS additive were introduced in the HTM solution, and a silica layer formed at the interface of $\mathrm{HTM}$ and $\mathrm{MAPbI}_{3}$ perovskite layers. Supplementary Figure 8 shows the $J-V$ curves of these corresponding devices, and no enhancement in $V_{\mathrm{OC}}$ and PCE was observed. This indicates that most of the trap states were located at the top surface and GBs of perovskite films, which agrees well with our previous study. ${ }^{32}$ Therefore, we attribute the $V_{\mathrm{OC}}$ enhancement in OS-wrapped perovskite devices to the upward shift of $E_{\mathrm{fn}}$ and thereby increased quasi-Fermi level splitting, which is believed to be induced by the reduction of trap states.

In addition to the improved photovoltaic performance, the stability of PSCs is dramatically enhanced by employing silicawrapped perovskite films. We first investigated the moisture stability of the OS-wrapped perovskites, and both the films and devices were exposed to ambient environment without encapsulation. After aging in air for 10 days, the impurity peak that is indexed to $\mathrm{PbI}_{2}$ appeared in the XRD pattern of the bare $\mathrm{MAPbI}_{3}$ film (Supplementary Figure 9a). In striking contrast, no impurity peak was identified in the XRD pattern of the $\mathrm{MAPbI}_{3} @ O$ OS film even after exposure to air for 60 days. The performance of the perovskite device employing a control $\mathrm{MAPbI}_{3}$ film degraded rapidly, as shown in Supplementary
Figure $9 \mathrm{~b}$. In comparison, the efficiency of the device made of the $\mathrm{MAPbI}_{3} @ \mathrm{OS}$ film maintained nearly 90\% of its original efficiency after $1200 \mathrm{~h}$. The significantly improved environmental stability can be attributed to the OS shells, which act as an effective physical barrier between the core perovskite materials and the ambience inhibiting the permeation of moisture and oxygen.

Apart from the enhanced moisture resistance by the OS coating, we speculate that reduction of trap states and suppressed ion migration in OS-coated perovskite films due to the passivation and blocking effect of the OS shell would contribute to enhancement of operational stability of the corresponding device. The mixed $\mathrm{FA}_{0.85} \mathrm{MA}_{0.15} \mathrm{~Pb}\left(\mathrm{I}_{0.85} \mathrm{Br}_{0.15}\right)_{3}$ perovskite films, which were demonstrated to deliver higher efficiency and more importantly to be more robust than $\mathrm{MAPbI}_{3}$ films under operation, were then employed to fabricate PSCs. FAMA is used to denote $\mathrm{FA}_{0.85} \mathrm{MA}_{0.15} \mathrm{~Pb}$ $\left(\mathrm{I}_{0.85} \mathrm{Br}_{0.15}\right)_{3}$ in the figures and following discussion. Figure 6a exhibits $J-V$ curves of the optimized PSCs based on thecontrol perovskite (FAMA) and OS-wrapped perovskite (FAMA@ OS). The control device with pristine FAMA perovskite yielded a high PCE of $19.8 \%$ with a $V_{\mathrm{OC}}$ of $1.10 \mathrm{~V}$. After OS coating, the PCE was boosted to $21.5 \%$ with a large $V_{\mathrm{OC}}$ of $1.15 \mathrm{~V}$ and a remarkable FF of $81.1 \%$ for the device fabricated with FAMA@OS, which proves the universality of the OS coating strategy. The corresponding photovoltaic parameters are summarized in Table 1 . The integrated $J_{S C}$ from the EQE spectrum shown in Figure $6 \mathrm{~b}$ reached $22.8 \mathrm{~mA} \mathrm{~cm}{ }^{-2}$, which agrees well with that from $J-V$ measurement. The steady-state 
photocurrent and efficiency measured at the maximum power point $(1.00 \mathrm{~V})$ are presented in Figure $6 c$, which confirms the device performance parameters extracted from the $J-V$ curve and verifies the absence of photocurrent hysteresis in our devices.

To examine whether the OS-wrapped perovskite can slow down degradation of the device, we performed stability tests of encapsulated PSCs based on FAMA perovskite films under full-spectrum simulated 1 sun illumination. The devices were encapsulated using a UV-curable resin inside of the glovebox before the subsequent operational stability testing in ambient air. Supplementary Figure 10 presents the schemes and images of a typical encapsulated perovskite device from both front view and back view. Each device was then electrically connected with a working load of $1000 \Omega$, and the $J-V$ scan was periodically recorded. As shown in Figure $6 \mathrm{~d}, \mathrm{e}$, both devices exhibited some loss in the first few hundred hours of operation followed by a remarkably stable period that lasted for thousands of hours. The initial period of steep efficiency loss is typically referred to as "burn-in", 48 and the trap states induced by photochemical reactions under illumination increase the energetic disorder in the system ${ }^{48,49}$ and thereby reduce the $V_{\mathrm{OC}}$ and $\mathrm{FF}$, and to a lesser extent $J_{\mathrm{SC}}$, leading to degradation in the encapsulated devices. Figure $6 \mathrm{~d}$ shows that the pristine FAMA device degraded very fast and lost $20 \%$ of its original efficiency within the first $168 \mathrm{~h}$. In striking contrast, the device with FAMA@OS had a significantly prolonged burn-in period of over $456 \mathrm{~h}$, during which $80 \%$ of its initial PCE was retained.

Moreover, the device with FAMA@OS also exhibited a much longer stable period after burn-in compared with the control FAMA device. This improvement primarily benefits from the retarded decay of photocurrent. The FAMA@OS device showed a lifetime of $5212 \mathrm{~h}$, which is defined by the duration to maintain $80 \%$ its efficiency after burn-in. ${ }^{21,50}$ This is nearly three times that of the control FAMA device ( 1776 h). Previous studies revealed that the iodide ion $\left(\mathrm{I}^{-}\right)$or $\mathrm{MA}^{+}$ might migrate throughout the films under illumination, which induces photocurrent hysteresis and accelerates device degradation. ${ }^{30,51-53}$ Because the activation energy $\left(E_{\mathrm{A}}\right)$ for ion migration at surfaces and GBs is roughly half that in the bulk, the surfaces and GBs of the perovskite grains are significant ion migration channels. ${ }^{53}$ Unlike the highly polymerized silica in previous work, ${ }^{40}$ the OS proposed in this work could enable the formation of a strong electrostatic interaction between the local negative charge surrounding the oxygen atoms (Lewis base sites in the OS layer) and the positive charge on the undercoordinated $\mathrm{Pb}$ at surfaces and GBs of the perovskite film. We speculate that such interaction may suppress the defect-mediated migration due to the effective passivation of these ionic defects in the OS-wrapped perovskite films, ${ }^{54}$ which will not only enhance the photovoltaic performance but more importantly prolong the operational stability of the corresponding devices. In addition, the presence of the OS layer as a physical barrier at the surfaces and GBs is also likely to inhibit ion diffusion either from the perovskite layer to CTLs or from the metal to perovskite layer, which prevents the formation of defects at the interfaces in the device. $^{55,56}$

The surpassing of $5000 \mathrm{~h}$ of operational stability under unfiltered simulated sunlight measured at operation conditions without a cooling device represents a significant advancement in the progress of perovskite photovoltaics. Our recent study shows that stability measurement at the $J_{S C}$ condition severely overestimates the device lifetime. ${ }^{57}$ We also want to point out that, though we did not control the temperature, the cell temperature remained at $60-65{ }^{\circ} \mathrm{C}$ during the measurement due to heating of the simulated sunlight. We understand that FAMA films generally have much poorer light stability than Cs-containing perovskites; however, the surpassing of $5000 \mathrm{~h}$ of operation lifetime using these low-stability perovskites shows the potential of this approach in enhancing the stability of perovskite photovoltaics.

In summary, we have demonstrated an OS-wrapped perovskite nanostructure exhibiting both significantly enhanced device efficiency and operational stability. With such a nanostructure, we observed a remarkably reduced tDOS as well as a prolonged carrier recombination lifetime, which indicates that the OS layer can efficiently passivate the defects at the surface and GBs. The optimized device with OSwrapped perovskite film delivered a decent PCE of $21.5 \%$ for $\mathrm{p}-\mathrm{i}-\mathrm{n}$ planar heterojunction PSCs. More importantly, the OS matrix that could block both external and internal degradation channels enabled all-around grain stabilization at the nanoscale, which not only increased the moisture resistivity but also greatly extended the device operational stability. We believe that the intrinsic instability of perovskite materials and devices is closely correlated with the defect states. The control of defects in perovskite materials and at the interfaces of perovskite devices is crucial for further improving the efficiency of PSCs to the theoretical limit and for intrinsically stabilizing perovskite-based materials and devices. This work represents a significant step in the development of highly efficient and intrinsically stable PSCs toward practical application. We anticipate that the synchronous defect passivation and grain stabilization will be an important direction in addressing the defect-driven intrinsic degradation in PSCs without sacrificing their photovoltaic performance, which can be easily extended to other perovskite optoelectronic applications such as photodetectors and light-emitting diodes.

\section{ASSOCIATED CONTENT}

\section{Supporting Information}

The Supporting Information is available free of charge on the ACS Publications website at DOI: 10.1021/acsenergylett.9b00608.

Details of device fabrication, measurements and characterization; tDOS analysis method; XRD patterns of perovskite films with and without OS; additional EDX spectra and ss-NMR spectra; schematic device structure; $J-V$ curves showing the hysteresis behavior of the OSwrapped perovskite device; steady-state PL spectra; ambient stability data; and tables providing weight ratios, photovoltaic parameters, efficiency comparisons, and fitting parameters (PDF)

\section{AUTHOR INFORMATION}

\section{Corresponding Author}

*E-mail: jhuang@unc.edu.

ORCID

Yang Bai: 0000-0001-8481-368X

Yun Lin: 0000-0001-6674-780X

Xiaolei Shi: 0000-0003-0905-2547

Zhi-Gang Chen: 0000-0002-9309-7993

Lianzhou Wang: 0000-0002-5947-306X 
Jinsong Huang: 0000-0002-0509-8778

\section{Notes}

The authors declare no competing financial interest.

\section{ACKNOWLEDGMENTS}

We acknowledge financial support from the Office of Naval Research under Award N00014-15-1-2713. Y.B. acknowledges financial support from a UQ Development Fellowship.

\section{REFERENCES}

(1) Huang, J.; Yuan, Y.; Shao, Y.; Yan, Y. Nat. Rev. Mater. 2017, 2 (7), 17042.

(2) Yang, W. S.; Park, B.-W.; Jung, E. H.; Jeon, N. J.; Kim, Y. C.; Lee, D. U.; Shin, S. S.; Seo, J.; Kim, E. K.; Noh, J. H.; Seok, S. I. Science 2017, 356 (6345), 1376-1379.

(3) Saliba, M.; Matsui, T.; Domanski, K.; Seo, J.-Y.; Ummadisingu, A.; Zakeeruddin, S. M.; Correa-Baena, J.-P.; Tress, W. R.; Abate, A.; Hagfeldt, A.; Grätzel, M. Science 2016, 354 (6309), 206-209.

(4) Tan, H.; Jain, A.; Voznyy, O.; Lan, X.; García de Arquer, F. P.; Fan, J. Z.; Quintero-Bermudez, R.; Yuan, M.; Zhang, B.; Zhao, Y.; Fan, F.; Li, P.; Quan, L. N.; Zhao, Y.; Lu, Z.-H.; Yang, Z.; Hoogland, S.; Sargent, E. H. Science 2017, 355, 722-726.

(5) Chen, H.; Ye, F.; Tang, W.; He, J.; Yin, M.; Wang, Y.; Xie, F.; Bi, E.; Yang, X.; Grätzel, M.; Han, L. Nature 2017, 550, 92.

(6) Christians, J. A.; Miranda Herrera, P. A.; Kamat, P. V. J. Am. Chem. Soc. 2015, 137 (4), 1530-1538.

(7) Wang, Q.; Chen, B.; Liu, Y.; Deng, Y.; Bai, Y.; Dong, Q.; Huang, J. Energy Environ. Sci. 2017, 10 (2), 516-522.

(8) Bryant, D.; Aristidou, N.; Pont, S.; Sanchez-Molina, I.; Chotchunangatchaval, T.; Wheeler, S.; Durrant, J. R.; Haque, S. A. Energy Environ. Sci. 2016, 9 (5), 1655-1660.

(9) Rehman, W.; McMeekin, D. P.; Patel, J. B.; Milot, R. L.; Johnston, M. B.; Snaith, H. J.; Herz, L. M. Energy Environ. Sci. 2017, $10,361-369$.

(10) Aristidou, N.; Eames, C.; Sanchez-Molina, I.; Bu, X.; Kosco, J.; Islam, M. S.; Haque, S. A. Nat. Commun. 2017, 8, 15218.

(11) Divitini, G.; Cacovich, S.; Matteocci, F.; Cinà, L.; Di Carlo, A.; Ducati, C. Nat. Energy 2016, 1, 15012.

(12) Han, Y.; Meyer, S.; Dkhissi, Y.; Weber, K.; Pringle, J. M.; Bach, U.; Spiccia, L.; Cheng, Y.-B. J. Mater. Chem. A 2015, 3 (15), 81398147.

(13) Yang, G.; Wang, C.; Lei, H.; Zheng, X.; Qin, P.; Xiong, L.; Zhao, X.; Yan, Y.; Fang, G. J. Mater. Chem. A 2017, 5, 1658-1666.

(14) Grancini, G.; Roldán-Carmona, C.; Zimmermann, I.; Mosconi, E.; Lee, X.; Martineau, D.; Narbey, S.; Oswald, F.; De Angelis, F.; Graetzel, M.; Nazeeruddin, M. K. Nat. Commun. 2017, 8, 15684.

(15) Hou, Y.; Du, X.; Scheiner, S.; McMeekin, D. P.; Wang, Z.; Li, N.; Killian, M. S.; Chen, H.; Richter, M.; Levchuk, I.; Schrenker, N.; Spiecker, E.; Stubhan, T.; Luechinger, N. A.; Hirsch, A.; Schmuki, P.; Steinrück, H.-P.; Fink, R. H.; Halik, M.; Snaith, H. J.; Brabec, C. J. Science 2017, 358 (6367), 1192-1197.

(16) Chen, P.; Bai, Y.; Wang, S.; Lyu, M.; Yun, J. H.; Wang, L. Adv. Funct. Mater. 2018, 28 (17), 1706923.

(17) You, J.; Meng, L.; Song, T.-B.; Guo, T.-F.; Yang, Y.; Chang, W.H.; Hong, Z.; Chen, H.; Zhou, H.; Chen, Q.; Liu, Y.; De Marco, N.; Yang, Y. Nat. Nanotechnol. 2016, 11 (1), 75-81.

(18) Arora, N.; Dar, M. I.; Hinderhofer, A.; Pellet, N.; Schreiber, F.; Zakeeruddin, S. M.; Grätzel, M. Science 2017, 358, No. 768.

(19) Bai, Y.; Dong, Q.; Shao, Y.; Deng, Y.; Wang, Q.; Shen, L.; Wang, D.; Wei, W.; Huang, J. Nat. Commun. 2016, 7, 12806.

(20) McMeekin, D. P.; Sadoughi, G.; Rehman, W.; Eperon, G. E.; Saliba, M.; Hörantner, M. T.; Haghighirad, A.; Sakai, N.; Korte, L.; Rech, B.; Johnston, M. B.; Herz, L. M.; Snaith, H. J. Science 2016, 351 (6269), 151-155.

(21) Wang, Z.; Lin, Q.; Chmiel, F. P.; Sakai, N.; Herz, L. M.; Snaith, H. J. Nat. Energy 2017, 2, 17135.
(22) Back, H.; Kim, G.; Kim, J.; Kong, J.; Kim, T. K.; Kang, H.; Kim, H.; Lee, J.; Lee, S.; Lee, K. Energy Environ. Sci. 2016, 9, 1258-1263. (23) Wolff, C. M.; Zu, F.; Paulke, A.; Toro, L. P.; Koch, N.; Neher, D. Adv. Mater. 2017, 29 (28), 1700159.

(24) Shao, Y.; Yuan, Y.; Huang, J. Nat. Energy 2016, 1, 15001.

(25) Zheng, X.; Chen, B.; Dai, J.; Fang, Y.; Bai, Y.; Lin, Y.; Wei, H.; Zeng, X. C.; Huang, J. Nat. Energy 2017, 2, 17102.

(26) Tress, W.; Yavari, M.; Domanski, K.; Yadav, P.; Niesen, B.; Correa Baena, J. P.; Hagfeldt, A.; Graetzel, M. Energy Environ. Sci. 2018, 11 (1), 151-165.

(27) Ahn, N.; Kwak, K.; Jang, M. S.; Yoon, H.; Lee, B. Y.; Lee, J.-K.; Pikhitsa, P. V.; Byun, J.; Choi, M. Nat. Commun. 2016, 7, 13422.

(28) Song, D.; Ji, J.; Li, Y.; Li, G.; Li, M.; Wang, T.; Wei, D.; Cui, P.; He, Y.; Mbengue, J. M. Appl. Phys. Lett. 2016, 108 (9), No. 093901.

(29) Khadka, D. B.; Shirai, Y.; Yanagida, M.; Miyano, K. J. Mater. Chem. C 2018, 6 (1), 162-170.

(30) Yin, W.-J.; Yang, J.-H.; Kang, J.; Yan, Y.; Wei, S.-H. J. Mater. Chem. A 2015, 3 (17), 8926-8942.

(31) Yin, W.-J.; Shi, T.; Yan, Y. Appl. Phys. Lett. 2014, 104 (6), No. 063903.

(32) Shao, Y.; Xiao, Z.; Bi, C.; Yuan, Y.; Huang, J. Nat. Commun. 2014, 5, 5784.

(33) Noel, N. K.; Abate, A.; Stranks, S. D.; Parrott, E. S.; Burlakov, V. M.; Goriely, A.; Snaith, H. J. ACS Nano 2014, 8 (10), 9815-9821.

(34) Zuo, L.; Guo, H.; deQuilettes, D. W.; Jariwala, S.; De Marco, N.; Dong, S.; DeBlock, R.; Ginger, D. S.; Dunn, B.; Wang, M.; Yang, Y. Sci. Adv. 2017, 3 (8), No. e1700106.

(35) Guerrero-Martinez, A.; Perez-Juste, J.; Liz-Marzan, L. M. Adv. Mater. 2010, 22 (11), 1182-1195.

(36) Stöber, W.; Fink, A.; Bohn, E. J. Colloid Interface Sci. 1968, 26 (1), 62-69.

(37) Selvan, S. T.; Tan, T. T.; Ying, J. Y. Adv. Mater. 2005, 17 (13), $1620-1625$.

(38) Wolcott, A.; Gerion, D.; Visconte, M.; Sun, J.; Schwartzberg, A.; Chen, S.; Zhang, J. Z. J. Phys. Chem. B 2006, 110 (11), 57795789.

(39) Sun, C.; Zhang, Y.; Ruan, C.; Yin, C.; Wang, X.; Wang, Y.; Yu, W. W. Adv. Mater. 2016, 28, 10088-10094.

(40) Liu, T.; Zhou, Y.; Li, Z.; Zhang, L.; Ju, M.-G.; Luo, D.; Yang, Y.; Yang, M.; Kim, D. H.; Yang, W.; Padture, N. P.; Beard, M. C.; Zeng, X. C.; Zhu, K.; Gong, Q.; Zhu, R. Adv. Energy Mater. 2018, 8 (22), 1800232.

(41) Askar, A. M.; Bernard, G. M.; Wiltshire, B.; Shankar, K.; Michaelis, V. K. J. Phys. Chem. C 2017, 121 (2), 1013-1024.

(42) Bi, C.; Wang, Q.; Shao, Y.; Yuan, Y.; Xiao, Z.; Huang, J. Nat. Commun. 2015, 6, 7747.

(43) Lin, Y.; et al. Adv. Mater. 2017, 29 (7), 1604545.

(44) Peng, J.; Khan, J. I.; Liu, W.; Ugur, E.; Duong, T.; Wu, Y.; Shen, H.; Wang, K.; Dang, H.; Aydin, E.; Yang, X.; Wan, Y.; Weber, K. J.; Catchpole, K. R.; Laquai, F.; De Wolf, S.; White, T. P. Adv. Energy Mater. 2018, 8 (30), 1801208.

(45) Walter, T.; Herberholz, R.; Müller, C.; Schock, H. J. Appl. Phys. 1996, 80 (8), 4411-4420.

(46) Melzer, C.; Koop, E. J.; Mihailetchi, V. D.; Blom, P. W. Adv. Funct. Mater. 2004, 14 (9), 865-870.

(47) Yang, J.-H.; Yin, W.-J.; Park, J.-S.; Wei, S.-H. Sci. Rep. 2015, 5, 16977.

(48) Mateker, W. R.; McGehee, M. D. Adv. Mater. 2017, 29 (10), 1603940.

(49) Peters, C. H.; et al. Adv. Mater. 2012, 24 (5), 663-668.

(50) Peters, C. H. Adv. Energy Mater. 2011, 1 (4), 491-494.

(51) Steirer, K. X.; Schulz, P.; Teeter, G.; Stevanovic, V.; Yang, M.; Zhu, K.; Berry, J. J. ACS Energy Lett. 2016, 1 (2), 360-366.

(52) Snaith, H. J.; Abate, A.; Ball, J. M.; Eperon, G. E.; Leijtens, T.; Noel, N. K.; Stranks, S. D.; Wang, J. T.-W.; Wojciechowski, K.; Zhang, W. J. Phys. Chem. Lett. 2014, 5 (9), 1511-1515.

(53) Yuan, Y.; Huang, J. Acc. Chem. Res. 2016, 49 (2), 286-293.

(54) Ran, C.; Xu, J.; Gao, W.; Huang, C.; Dou, S. Chem. Soc. Rev. 2018, 47 (12), 4581-4610. 
(55) Christians, J. A.; Schulz, P.; Tinkham, J. S.; Schloemer, T. H.; Harvey, S. P.; Tremolet de Villers, B. J.; Sellinger, A.; Berry, J. J.; Luther, J. M. Nat. Energy 2018, 3 (1), 68-74.

(56) Domanski, K.; Alharbi, E. A.; Hagfeldt, A.; Grätzel, M.; Tress, W. Nat. Energy 2018, 3 (1), 61-67.

(57) Lin, Y.; Chen, B.; Fang, Y.; Zhao, J.; Bao, C.; Yu, Z.; Deng, Y.; Rudd, P. N.; Yan, Y.; Yuan, Y.; Huang, J. Nat. Commun. 2018, 9 (1), 4981. 